\title{
CONHECIMENTO DE PROFISSIONAIS DE ENFERMAGEM SOBRE HIGIENIZAÇÃO DAS MÃOS
}

\author{
Flávia Maria Derhun ${ }^{1}$, Verusca Soares de Souza ${ }^{2}$, Maria Antônia Ramos Costa ${ }^{3}$, Kelly Cristina Inoue ${ }^{4}$, Laura \\ Misue Matsuda ${ }^{5}$
}

RESUMO: Estudo analítico, de abordagem quantitativa, realizado no mês de janeiro de 2014 e que teve como objetivo analisar o conhecimento de profissionais de enfermagem sobre higienização das mãos. Participaram 267 trabalhadores, de dois hospitais da região noroeste do Paraná, que preencheram um questionário adaptado do Ministério da Saúde, intitulado Teste de Conhecimento a Respeito da Higienização das Mãos para Profissionais de Saúde, contendo oito questões. Os dados foram tratados por meio dos programas Statistical Package for Social Sciences 20 (SPSS) e Epi Info ${ }^{T M}$ 7.1.3. Constatou-se percentual elevado de acertos das questões, exceto naquelas que se referiam à Rota de infecção e Tempo mínimo de fricção das mãos com preparação alcoólica. Concluiu-se que $86,52 \%$ dos profissionais investigados não conheciam na íntegra as instruções para higienização das mãos. Sugere-se que o tema higienização das mãos seja abordado com as equipes investigadas, por meio de processos educativos permanentes, pautados em metodologias ativas.

DESCRITORES: Pessoal de saúde; Segurança do paciente; Controle de infecções; Higiene das mãos; Enfermagem.

\section{KNOWLEDGE OF NURSING PROFESSIONALS REGARDING HAND HYGIENE}

\begin{abstract}
This quantitative analytical study, performed in January 2014, aimed to analyze the knowledge of nursing professionals regarding hand hygiene. Participants were 267 employees of two hospitals in the northwest of Paraná, who filled out an adapted questionnaire of the Ministry of Health, entitled Knowledge Test Regarding Hand Hygiene for Health Professionals, which contains eight questions. The data were processed using the Statistical Package for the Social Sciences 20 (SPSS) and Epi InfoTM 7.1.3. A high percentage of correct responses to the questions was found, except for those that referred to the Route of infection and Minimum rubbing time of hands with an alcohol preparation. It was concluded that $86.52 \%$ of the investigated professionals did not fully know the instructions for hand hygiene. It is suggested that the hand hygiene issue be addressed with the teams investigated through permanent educational processes, guided by active methods.
\end{abstract}

DESCRIPTORS: Health personnel; Patient safety; Infection control; Hand hygiene; Nursing.

\section{CONOCIMIENTO DE PROFESIONALES DE ENFERMERÍA ACERCA DE LA HIGIENIZACIÓN DE LAS MANOS}

RESUMEN: Estudio analítico, de abordaje cuantitativo, realizado en el mes de enero de 2014 cuyo objetivo fue analizar el conocimiento de profesionales de enfermería acerca de la higienización de las manos. Participaron 267 trabajadores, de dos hospitales de la región noroeste de Paraná, que completaron un cuestionario adaptado del Ministerio de la Salud, intitulado Test de Conocimiento Acerca de la Higienización de las Manos para Profesionales de Salud, con ocho cuestiones. Los datos fueron sometidos a los programas Statistical Package for Social Sciences 20 (SPSS) y Epi Info ${ }^{T M}$ 7.1.3. Se constató percentual elevado de aciertos de las cuestiones, excepto las que se referían a la Ruta de infeccióny Tiempo mínimo de fricción de las manos con preparación alcohólica. Se concluyó que $86,52 \%$ de los profesionales investigados no conocían integralmente las instrucciones para higienización de las manos. Se sugiere que el tema higienización de las manos sea abordado con los equipos investigados, por medio de procesos educativos permanentes, basados en metodologías activas.

DESCRIPTORES: Personal de salud; Seguridad del paciente; Control de infecciones; Higiene de las manos; Enfermería.

${ }^{1}$ Enfermeira. Mestranda em Enfermagem. Universidade Estadual de Maringá. Maringá, PR, Brasil.

2Enfermeira. Mestre em Enfermagem. Docente de Enfermagem da Universidade Estadual do Paraná. Paranavaí, PR, Brasil.

${ }^{3}$ Enfermeira. Doutora em Enfermagem. Docente de Enfermagem da Universidade Estadual do Paraná. Paranavaí, PR, Brasil.

${ }^{4}$ Enfermeira. Doutora em Enfermagem. Docente de Enfermagem e Medicina da Faculdade Ingá. Maringá, PR, Brasil.

${ }^{5}$ Enfermeira. Doutora em Enfermagem Fundamental. Docente da graduação e Pós-Graduação em Enfermagem da Universidade Estadual de Maringá. Maringá, PR, Brasil.

Autor Correspondente:

Verusca Soares de Souza

Universidade Estadual do Paraná

Av. Gabriel Espiridião, S/N - 87700-000 - Paranavaí, PR, Brasil

E-mail:veruscasoares@gmail.com
Recebido: 29/02/2016

Finalizado: 18/09/2016 


\section{INTRODUÇÃO}

A higienização das mãos é uma ação simples, rápida e fácil de ser realizada. Além disso, é uma medida individual, primária e imprescindível para a prevenção e controle de Infecções Relacionadas à Assistência à Saúde (IRAS).

A importância da higiene das mãos tomou vulto a partir de 1846, quando o médico húngaro Ignaz Phillip Semmelweis relacionou a incidência de febre puerperal com a precária higienização das mãos ${ }^{(1)}$. Desde então, os avanços na ciência e na tecnologia, associados com a melhoria das condições de vida, têm facilitado a prática de higiene das mãos, a qual consiste numa das principais medidas à prevenção de IRAS ${ }^{(2-3)}$.

A higienização das mãos dos profissionais de saúde, realizada de forma cuidadosa e frequente, relaciona-se diretamente com a segurança do paciente por levar à ruptura do elo de transmissão de patógenos ${ }^{(1,4)}$. Isto porque a falta de higienização adequada das mãos dos profissionais contribui para o desenvolvimento de IRAS, que se constituem em eventos adversos advindos do processo de cuidar; e, não raras vezes, resultam em aumento de custos assistenciais, do período de internação e também das taxas de morbidade e de mortalidade ${ }^{(1,3)}$.

As mãos dos profissionais de saúde, em especial da equipe de enfermagem, por manter contato direto e frequente com o paciente, são fontes e veículo de disseminação de patógenos. Nesse aspecto, pesquisa ${ }^{(5)}$ realizada com 48 profissionais de saúde de diferentes áreas e que atuavam em um hospital privado de Itumbiara, estado de Goiás, constatou que a contagem da microbiota das mãos dos profissionais da equipe de enfermagem durante a atividade laboral possuía alto índice de contaminação, inclusive de microrganismos multirresistentes.

Apesar das evidências científicas e das disposições legais, a literatura ${ }^{(6-9)}$ aponta que grande parte dos profissionais que prestam assistência à saúde não adotam as recomendações mencionadas. Dentre as justificativas, constam o esquecimento, a falta de tempo, a distância da pia/lavabo, a irritação da pele, bem como a falta de informações sobre o impacto da higienização das mãos nas taxas das IRAS ${ }^{(1,10)}$.

Embora a higienização das mãos seja um procedimento simples e até corriqueiro, a falta de conhecimento/informação sobre os riscos de não realizar, ou de realizá-la incorretamente, são fatores que podem e devem ser sanados. Desse modo, o presente estudo se justifica porque a higienização das mãos é uma das seis medidas adotadas pela Agência Nacional de Vigilância Sanitária (ANVISA) à promoção da segurança do paciente. Além disso, a obtenção e divulgação de dados atuais podem contribuir para o estabelecimento de ações eficazes, relacionadas à prevenção e/ou redução de IRAS, especificamente na área da saúde.

Com o interesse voltado à segurança do paciente e também dos trabalhadores que atuam diretamente nos processos de cuidado, questiona-se: os profissionais de enfermagem conhecem, na íntegra, as instruções referentes à higienização das mãos? Para responder a esse questionamento, este estudo tem como objetivo analisar o conhecimento de profissionais de duas equipes de enfermagem, acerca da higienização das mãos.

\section{- MÉTODO}

Estudo analítico, de abordagem quantitativa, realizado no mês de janeiro de 2014, em um hospital público de ensino e em um hospital próprio, de operadora de plano privado de saúde, ambos situados na região noroeste do Paraná, aqui denominados de Hospital A e Hospital B.

No hospital A, o quadro da enfermagem era composto por 295 profissionais, sendo 89 enfermeiros e 206 técnicos de enfermagem. No hospital B, 33 profissionais de enfermagem, dos quais sete eram enfermeiros e 26 técnicos de enfermagem. Apenas o hospital A dispunha de Serviço de Educação Contínua.

A coleta dos dados foi realizada por meio de censo e foram inclusos no estudo todos os profissionais que trabalhavam há mais de três meses na instituição e exclusos os profissionais que estavam de férias 
ou atestado médico no período de coleta de dados. Participaram 240 profissionais de enfermagem do hospital A (81,4\% do quadro funcional de enfermagem da instituição) e 27 do hospital B (81,8\% do quadro funcional de enfermagem da instituição); totalizando 267 participantes que corresponderam a $81,6 \%$ do total de profissionais de ambas instituições.

Durante o turno de trabalho de cada profissional, em local privativo, após autorização formal, os participantes responderam a um questionário composto de duas partes. A primeira era destinada à obtenção de dados de caracterização, e a segunda, parte específica, possuía oito questões, extraídas do questionário denominado Teste de Conhecimento a Respeito da Higienização das Mãos para Profissionais de Saúde ${ }^{(11)}$.

Devido à falta de parâmetro para avaliação do conhecimento dos profissionais de saúde com a utilização do instrumento antes referido, adotou-se o Índice de Positividade (IP), que é interpretado a partir do número de respostas positivas (corretas), da seguinte forma: Desejável (100\% de positividade); Adequado (99\% a 90\% de positividade); Seguro (89\% a $80 \%$ de positividade); Limítrofe (79\% a $71 \%$ de positividade); e Sofrível (70\% ou menos de positividade) $)^{(12-13)}$.

Os dados foram compilados em planilhas eletrônicas e após, foi realizada análise estatística descritiva e inferencial, com os programas Statistical Package for Social Sciences 20 (SPSS) e Epi Info ${ }^{\text {TM 7.1.3. }}$

Para as variáveis contínuas (idade, tempo de trabalho em Enfermagem e tempo de trabalho na instituição), foi verificado o valor mínimo, máximo, média e desvio padrão (DP). Para as variáveis categóricas (sexo, categoria profissional, respostas corretas de cada questão), foram calculadas as frequências e porcentagens.

Considerando-se significância estatística quando $p$-valor $\leq 0,05$, procedeu-se o teste de associação (teste qui-quadrado (x2) corrigido e o Teste Exato de Fisher) entre a quantidade de acertos de cada questão com as seguintes variáveis de interesse: categoria profissional e tempo de trabalho na enfermagem. Para isso, a variável tempo de trabalho na enfermagem foi dicotomizada de acordo com a sua mediana.

O presente estudo foi realizado em consonância com a legislação ética vigente ${ }^{(14)}$ e o seu Projeto de Pesquisa foi aprovado pelo Comitê Permanente de Ética em Pesquisas Envolvendo Seres Humanos da Universidade Estadual de Maringá, sob Parecer nº 462.941/2013.

\section{- RESULTADOS}

Participaram 31(11,6\%) homens e $236(88,4 \%)$ mulheres, cuja idade variou entre 21 a 70 anos (média $=41,5$ anos; DP=10,2anos). O tempo de atuação na enfermagem se situou entre um a 26 anos (média=16,6 anos; DP=9,4 anos) e o tempo de trabalho no hospital, de um mês a 30 anos (média=9,9 anos; $\mathrm{DP}=7,9$ anos).

Dos profissionais, 241 (90,3\%) afirmaram ter recebido algum treinamento sobre higienização das mãos. Em relação à preparação alcoólica para fricção das mãos, 262 (98,1\%) referiram saber da disponibilidade/existência na instituição.

Na Tabela 1 está contida a distribuição das respostas corretas, sua associação com categoria profissional e tempo de trabalho na enfermagem, bem como a classificação do grau de conhecimento de acordo com o IP.

Na Tabela 2 consta a distribuição de frequências e porcentagens das questões de múltipla escolha que abordavam a técnica de higienização das mãos pelos participantes. 
Tabela 1 - Associação entre acertos às questões sobre higienização das mãos, categoria profissional e tempo de serviço. Paranavaí-PR, Maringá-PR, Brasil, 2014

\begin{tabular}{|c|c|c|c|c|c|c|c|c|}
\hline \multirow[t]{3}{*}{ Questão } & \multicolumn{3}{|c|}{ Categoria profissional } & \multicolumn{3}{|c|}{ Tempo de trabalho } & \multirow[t]{3}{*}{ Total } & \multirow{3}{*}{ IP } \\
\hline & ENF & TE & \multirow[t]{2}{*}{ p-valor } & $<7$ anos & $>7$ anos & \multirow[t]{2}{*}{ p-valor } & & \\
\hline & $\%$ & $\%$ & & $\%$ & $\%$ & & & \\
\hline Rota de transmissão cruzada & 98,9 & 93,7 & $0,0631^{a}$ & 98,5 & 92,7 & $0,0352^{a}$ & 95,5 & Adequado \\
\hline Fonte mais frequente para IRAS & 39,1 & 48,3 & $0,1792^{\mathrm{b}}$ & 41,5 & 48,9 & $0,2776^{\mathrm{b}}$ & 45,3 & Sofrível \\
\hline $\begin{array}{l}\text { Tempo mínimo de fricção com } \\
\text { preparação alcoólica }\end{array}$ & 45,7 & 31,4 & $0, \mathbf{0 3 0 6}^{\mathrm{b}}$ & 43,8 & 29,2 & $0,0183^{b}$ & 36,3 & Sofrível \\
\hline $\begin{array}{l}\text { Cobertura das mãos com } \\
\text { preparação alcoólica }\end{array}$ & 100 & 94,9 & $0, \mathbf{0 2 9 8}^{\mathrm{a}}$ & 97,7 & 95,6 & $0,5017^{\mathrm{a}}$ & 96,6 & Adequado \\
\hline $\begin{array}{l}\text { Mãos secas antes do uso da } \\
\text { preparação alcoólica }\end{array}$ & 88 & 82,9 & $0,3480^{\mathrm{b}}$ & 81,5 & 87,6 & $0,2295^{b}$ & 84,6 & Seguro \\
\hline $\begin{array}{l}\text { Uso de papel toalha após } \\
\text { a fricção com preparação } \\
\text { alcoólica }\end{array}$ & 93,5 & 88,6 & $0,2855^{b}$ & 87,7 & 92,7 & $0,2407^{b}$ & 90,3 & Adequado \\
\hline
\end{tabular}

TE=Técnico de Enfermagem; ENF=Enfermeiro; ${ }^{a}$ Teste Exato de Fisher; ${ }^{\mathrm{b}}$ Teste $\mathrm{x} 2$ corrigido.

Tabela 2 - Distribuição de frequências e porcentagens das respostas às questões de múltipla escolha. ParanavaíPR, Maringá-PR, Brasil, 2014

\section{Questões e alternativas de resposta}

n

$\%$

Qual é a principal rota de transmissão cruzada de microrganismos potencialmente

patogênicos entre pacientes em serviços de saúde?

\begin{tabular}{lcc}
\hline a) Mãos do profissional de saúde quando não estão higienizadas & 255 & 95,5 \\
\hline b) Circulação do ar no hospital & 10 & 3,7 \\
\hline c) Exposição do paciente a superfícies colonizadas & 0 & 0 \\
\hline d) Compartilhar objetos não invasivos & 2 & 0,8 \\
\hline
\end{tabular}

Qual é a fonte de microrganismos mais frequente responsável pelas infecções relacionadas à assistência à saúde?

\begin{tabular}{lcc}
\hline a) Microrganismos no sistema de água do hospital & 1 & 0,4 \\
\hline b) Microrganismos no ar do hospital & 3 & 1,1 \\
\hline c) Microrganismos já presentes no paciente ou nas proximidades dele & 121 & 45,3 \\
\hline d) Microrganismos no ambiente hospitalar & 142 & 53,2 \\
\hline
\end{tabular}

Qual é o tempo mínimo necessário para a preparação alcoólica destruir a maioria dos microrganismos presentes em suas mãos?

\begin{tabular}{lll}
\hline a) 3 segundos & 14 & 5,2 \\
\hline b) 10 segundos & 75 & 28,1 \\
\hline c) 20 segundos & 97 & 36,3 \\
\hline d) 1 minuto & 81 & 30,4
\end{tabular}

\section{- DISCUSSÃO}

O percentual de respostas corretas foi "adequado" no que se refere à rota de transmissão cruzada $(95,5 \%)$, cobertura das mãos com preparação alcoólica $(96,6 \%)$ e uso de papel toalha após a fricção com preparação alcoólica (90,3\%). Foi "seguro" para a questão sobre a necessidade de as mãos estarem secas antes do uso da preparação alcoólica (84,6\%). Tais resultados foram positivos, contudo, ainda existe espaço para aprimoramento dos profissionais de enfermagem sobre esses aspectos, porque a higienização é uma das estratégias mais eficientes para o controle das IRAS; e por isso, deve ser realizada da forma mais correta possível ${ }^{(1-2,4,6)}$. 
Em contrapartida, obteve-se percentual "sofrível" de acertos para as questões relacionadas à fonte mais frequente de IRAS (45,3\%) e tempo mínimo de destruição de microrganismos com preparação alcoólica (36,3\%). No que tange à fonte mais frequente para IRAS, observou-se que mais da metade dos participantes mencionaram, equivocadamente, que esta era representada pelos microrganismos presentes no ambiente hospitalar (53,2\%). Embora os microrganismos presentes no hospital possam causar IRAS, a fonte mais frequente são os microrganismos alojados no paciente ou nas suas proximidades $^{(3,11)}$.

Dada a importância da equipe de enfermagem no controle e prevenção de transmissão de microorganismos com maior potencial à ocorrência de IRAS, como aqueles presentes no paciente, a abordagem dessa temática, apesar de gerar descrença em muitos profissionais, deve se constituir em elemento chave no planejamento e no estabelecimento de medidas preventivas ${ }^{(3)}$.

Quanto ao tempo mínimo de fricção antisséptica das mãos, 81 (30,3\%) participantes apontaram que um minuto seria o tempo necessário para destruir a maioria dos microrganismos com a preparação alcoólica. Destaca-se que é recomendada a fricção antisséptica das mãos por, no mínimo, 20 a 30 segundos $^{(1-2,15)}$. Nesse sentido, pondera-se que apesar de um minuto não ser o tempo mínimo preconizado, se durante a rotina assistencial, estes profissionais friccionarem as mãos por esse período, estarão exercendo influência positiva no controle das IRAS.

Em contrapartida, alerta-se ao fato de uma parcela importante dos profissionais (33,3\%) ter indicado períodos inferiores ao tempo mínimo de fricção recomendado. Ao assumir que as mãos devem ser friccionadas, de acordo com a técnica básica de higiene, até secagem completa da preparação a base de álcool, os profissionais podem ter sido induzidos ao erro pela utilização de quantidade insuficiente do produto para cobertura total da mão(1).

Ao comparar a quantidade de acertos das respostas com a categoria profissional, constatou-se diferença significativa entre enfermeiros e técnicos de enfermagem nas questões sobre o tempo mínimo de fricção com preparação alcoólica ( $p$-valor=0,0306) e a necessidade de cobertura total das mãos com preparação alcoólica ( $p$-valor=0,0298). Esse resultado reafirma o fato de que o diferencial entre essas categorias se encontra no embasamento teórico requerido para o exercício profissional. Ademais, esses dados coadunam com os resultados de um estudo ${ }^{(6)}$ desenvolvido por meio da observação de 369 profissionais de saúde em Unidades Básicas de Saúde, de dez municípios da Região Sul de Santa Catarina. Constatou-se que, em relação à higienização das mãos, os profissionais de nível superior detêm maior conhecimento do que aqueles que possuem nível médio ou técnico.

Apesar de não apresentar significância estatística, chama atenção o resultado da questão que se refere à fonte mais frequente para IRAS, cuja frequência de acertos foi maior entre os técnicos de enfermagem. Isso é preocupante, pois o enfermeiro como líder de equipe precisa ter habilidades para a supervisão da assistência de enfermagem, tendo em vista ações que contribuam a segurança do paciente $^{(16)}$.

Nessa perspectiva, destaca-se que o enfermeiro deve orientar e monitorar as práticas de higiene das mãos seja da equipe de saúde, dos pacientes e também dos familiares, mas se o seu conhecimento for limitado, o procedimento tende a ser ignorado e/ou equivocado por todos. Para minimizar esse tipo de situação, sugere-se que os enfermeiros dos hospitais investigados se apropriem das orientações pertinentes à higienização das mãos e as realizem corretamente, em consonância com o que é preconizado pelos órgãos oficiais ${ }^{(1-2,15)}$.

Sobre a quantidade de acertos às questões específicas de acordo com o tempo de atuação na enfermagem, verificou-se diferença significativa na questão que indaga sobre a principal rota de transmissão cruzada em serviços de saúde ( $p$-valor=0,0352) e naquela que se refere ao tempo mínimo necessário para a preparação alcoólica destruir a maioria dos microrganismos presentes nas mãos ( $p$-valor=0,0183). Essas duas questões obtiveram mais acertos dentre aqueles que possuíam menor tempo de atuação profissional. Nesse sentido, infere-se que entre aqueles com menos tempo de trabalho, o conhecimento tende a ser mais atualizado devido ao egresso recente dos cursos de formação, pois, no Brasil, a higienização das mãos com preparação alcoólica teve impulso nos últimos anos, e a sua disponibilização tornou-se obrigatória nos serviços de saúde somente em $2010^{(17)}$.

No entanto, em uma investigação( ${ }^{(6)}$ conduzida em Unidades Básicas de Saúde, com objetivo de 
avaliar a qualidade da higienização das mãos, antes de procedimentos cirúrgicos, não se observou diferença significativa entre maior e menor tempo de formação na área de atuação. Mas a variável idade, que pode refletir o tempo de formação, mostrou que $47,4 \%$ dos profissionais com mais de 35 anos de idade realizavam-na corretamente, enquanto que entre profissionais mais jovens o percentual era de apenas $36,6 \%$.

A higienização das mãos é a medida mais importante para o controle de infecção cruzada $^{(9)} \mathrm{e}$ isso há muito tempo tem sido divulgado entre os profissionais da saúde. Contudo, no que se refere à rota de transmissão cruzada, novamente se observou que os profissionais com maior tempo de atuação demonstraram menor nível de conhecimento ( $p$-valor $=0,0352$ ) do que os demais profissionais da equipe, denotando a importância da implantação de um processo de educação permanente nas instituições de saúde, com a finalidade de manter a equipe constantemente atualizada sobre as rotinas e procedimentos necessários ao desempenho de suas funções ${ }^{(1,18)}$.

As lacunas de conhecimento apresentadas em relação ao uso da preparação alcoólica podem ser justificadas, em parte, pelo fato de que a higienização simples com água e sabão ainda é o meio preferido pelos profissionais de saúde ${ }^{(7,18)}$. Apesar de a higiene das mãos com preparação alcoólica ser um procedimento fácil e rápido, indicado para quando as mãos não estiverem visivelmente sujas, antes e depois de tocar o paciente ou após a remoção das luvas ${ }^{(15)}$, observa-se que essa conduta pode não estar sendo realizada sistematicamente, em detrimento da falta de sensibilização e/ou de conhecimento do profissional.

Destaca-se, pelos resultados obtidos, a necessidade de estabelecimento e/ou intensificação das estratégias educativas e de monitoramento de indicadores, para ampliação do conhecimento das equipes de enfermagem e realização da higienização das mãos de forma adequada, seja em termos de frequência e/ou técnica. Isso porque, apesar de os profissionais da enfermagem reconhecerem a importância da higienização das mãos para o controle de IRAS, o discurso nem sempre se alinha à prática.

Ao exemplo da afirmativa anterior, um estudo ${ }^{(19)}$ conduzido em uma Unidade de Terapia Intensiva (UTI) Adulto, com 49 profissionais da saúde, incluindo profissionais de enfermagem, médicos e fisioterapeutas, apontou que, após a prestação da assistência, o momento em que os profissionais mais realizaram o procedimento foi quando mantiveram contato com o paciente, dando preferência apenas à sua própria segurança.

Considera-se necessário implantar estratégias para a higienização das mãos ${ }^{(11)} \operatorname{como}$ acesso fácil às preparações alcoólicas e demais insumos destinados a esse fim; educação dos profissionais; fixação de lembretes visuais em pontos estratégicos; e monitoração das práticas de higienização das mãos com retorno do desempenho aos profissionais. Ações dessa natureza tendem a melhorar a adesão global à realização da referida prática ${ }^{(18-19)}$. Nessa perspectiva, destaca-se que, eletronicamente, existe um guia para a implantação da estratégia multimodal, elaborado pela Organização Mundial de Saúde ${ }^{(11)}$, para a melhoria da higienização das mãos.

A saber, na estratégia referida, são incluídos elementos chave como a instrução da equipe e programas de motivação; adoção de produtos alcoólicos como o padrão ouro; uso de indicadores de desempenho; e o forte comprometimento de todas as partes interessadas, tais como equipe da linha de frente, gerentes e líderes de saúde ${ }^{(1,3)}$. Medidas desse tipo devem ser encorajadas porque, neste estudo, apesar de 241 (90,3\%) participantes terem recebido treinamento em higienização das mãos, os resultados apontam que existem lacunas no conhecimento dessa temática pelas equipes investigadas, principalmente no que se refere ao tempo de fricção das mãos com preparação alcoólica.

\section{- CONCLUSÃO}

Os profissionais de enfermagem tiveram alto percentual de acertos nas questões sobre higienização das mãos. No entanto, $86,5 \%$ dos profissionais investigados não conheciam na íntegra as instruções para higienização das mãos. Para que haja melhoria em alguns quesitos, sugere-se que as instituições implementem medidas voltadas à educação permanente dos seus trabalhadores, e que a higienização das mãos seja um tema de maior prioridade. 
Sugere-se que sejam realizados, posteriormente, estudos observacionais que avaliem a qualidade e a taxa de adesão dos trabalhadores ao procedimento de higienização das mãos, uma vez que, neste censo, o objetivo foi analisar o conhecimento dos profissionais, o que não possibilita afirmar que o conhecimento é aplicado à prática assistencial.

Espera-se que este estudo, no campo científico, ofereça subsídios para outras pesquisas com desenhos mais robustos; e no campo da prática, produza reflexões e quiçá, aumente a adesão dos profissionais de saúde à prática da higienização sistemática e correta das mãos.

As principais limitações deste estudo se encontram no fato de não ter incluído outras categorias profissionais da área da saúde e por não ter avaliado o conhecimento dos participantes por meio da observação da técnica. Desse modo, sugerem que novos estudos sejam realizados junto aos demais profissionais de saúde, com foco no conhecimento teórico, mas principalmente, em sua aplicação na prática assistencial.

\section{AGRADECIMENTOS}

Ao CNPq pela bolsa de Iniciação Científica concedida.

\section{REFERÊNCIAS}

1. Agência Nacional de Vigilância Sanitária (BR). Manual de Segurança do Paciente: Higienização das mãos. Brasília (DF): ANVISA; 2010.

2. World Health Organization. WHO guidelines on hand hygiene in health care. First global patient safety challenge: clean care is safe care. Geneva (SUI): WHO; 2009.

3. Agência Nacional de Vigilância Sanitária (BR). Segurança do Paciente e Qualidade em Serviços de Saúde: Medidas de Prevenção de Infecção Relacionada à Assistência à Saúde. Brasília (DF): ANVISA; 2013.

4. Krummenauer EC, Adam MS, Muller LB, Machado JAA, Carneiro M. Are awareness strategies effective in improving adherence to hand hygiene in health care?. J Infect Control. 2013; 2(2): 126-7.

5. Custódio J, Alves JF, Silva FM, Dolinger EJO, dos Santos JGS, de Brito DVD. Avaliação microbiológica das mãos de profissionais de saúde de um hospital particular de Itumbiara. Rev. ciênc. méd. 2009; 18(1): 7-11.

6. Locks L, Lacerda JT, Gomes E, Serratine ACP. Qualidade da higienização das mãos de profissionais atuantes em unidades básicas de saúde. Rev. Gaúcha Enferm. 2011; 32(3): 569-75.

7. Borges Primo MG, Ribeiro LCM, Figueiredo LFS, Sirico SCA, de Souza MA. Adesão à prática de higienização das mãos por profissionais de saúde de um Hospital Universitário. Rev. Eletr. Enf. [Internet] 2010; 12(2) [acesso em 05 set 2014]. Disponível: http://www.fen.ufg.br/revista/v12/n2/v12n2a06.htm.

8. Martinez MR, Campos LAAF, Nogueira PCK. Adesão à técnica de lavagem de mãos em Unidade de Terapia Intensiva Neonatal. Rev. paul. pediatr. 2009; 27(2): 179-85.

9. Rezende KCAD, Tipple AFV, Siqueira KM, Alves SB, Salgado TA, Pereira MS. Adesão à higienização das mãos e ao uso de equipamentos de proteção pessoal por profissionais de enfermagem na atenção básica em saúde. Ciênc Cuid Saude. 2012; 11(2): 343-51.

10. Oliveira AC, Cardoso CS, Mascarenhas D. Precauções de contato em Unidade de Terapia Intensiva: fatores facilitadores e dificultadores para a adesão dos profissionais. Rev. esc. enferm. USP. 2010; 44(1): 161-5.

11. Organização Mundial da Saúde (OMS). Guia Para Implementação: Um Guia para a implantação da estratégia multimodal da OMS para a melhoria da higienização das mãos a observadores: estratégia multimodal da OMS para a melhoria da higienização das mãos. Brasília (DF): Organização Pan-Americana da Saúde, Agência Nacional de Vigilância Sanitária; 2008.

12. Haddad MCL, Évora YDM. Qualidade da assistência de enfermagem: a opinião do paciente internado em 
hospital universitário público. Cienc Cuid Saude. 2008; 7(Suppl 1): 45-52.

13. Nonino EAPM, Anselmi ML, Dalmas JC. Qualidade do procedimento curativo em pacientes internados em um hospital universitário. Rev Latino-am Enfermagem. [Internet] 2008; 16(1) [acesso em 2 nov 2014]. Disponível: http://dx.doi.org/10.1590/S0104-11692008000100010.

14. Ministério da Ministério da Saúde (BR). Conselho Nacional de Saúde. Diretrizes e normas regulamentadoras de pesquisa envolvendo seres humanos. Resolução n. 466, de 12 de dezembro de 2012. Brasília; 2012.

15. Ministério da Saúde (Brasil). Portaria n. 1.377, de 9 de julho de 2013. Aprova os protocolos de segurança do paciente. Brasília (DF); 2013. [Anexo 01: Protocolo para a prática de higiene das mãos em serviços de saúde].

16. Inoue KC, Matsuda LM. Segurança do paciente: abordando um antigo problema. Cienc Cuid Saude. 2013; 12(2): 208-9.

17. Agência Nacional de Vigilância Sanitária (Brasil). RDC n. 42, de 25 de outubro de 2010. Dispõe sobre a obrigatoriedade de disponibilização de preparação alcoólica para fricção antisséptica das mãos, pelos serviços de saúde do País, e dá outras providências. Brasília (DF): Diário Oficial União, 2010. Seção 1.

18. do Prado MF, Oliveira ACJ, do Nascimento TMB, de Melo WA, do Prado DB. Estratégia de promoção à higienização das mãos em unidade de terapia intensiva. Cienc cuid saude. 2012; 11(3): 557-64.

19. Bathke J, Cunico PA, Maziero ECS, Cauduro FLF, Sarquis LMM, Cruz EDA. Infraestrutura e adesão à higienização das mãos: desafios à segurança do paciente. Rev. Gaúcha Enferm. 2013; 34(2): 78-85. 